ARTICLE OPEN

\title{
On the use of CdSe scintillating nanoplatelets as time taggers for high-energy gamma detection
}

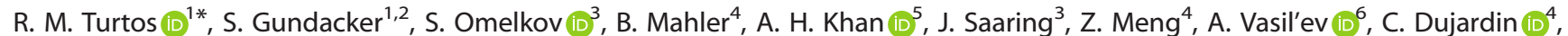 \\ M. Kirm ${ }^{3}$, I. Moreels $\mathbb{1 D}^{5}$, E. Auffray ${ }^{1}$ and P. Lecoq ${ }^{1}$
}

The technological challenge imposed by the time resolution essential to achieve real-time molecular imaging calls for a new generation of ultrafast detectors. In this contribution, we demonstrate that CdSe-based semiconductor nanoplatelets can be combined with standard scintillator technology to achieve 80 ps coincidence time resolution on a hybrid functional pixel. This result contrasts with the fact that the overall detector light output is considerably affected by the loss of index-light-guiding. Here, we exploit the principle of $511 \mathrm{keV}$ energy sharing between a high-Z, high stopping power bulk scintillator, and a nano-scintillator with sub-1 ns radiative recombination times, aiming at a breakthrough in the combined energy and time resolution performance. This proof-of-concept test opens the way to the design and study of larger size sensors using thin nanocomposite layers able to perform as efficient time taggers in a sampling detector geometry of new generation.

npj 2D Materials and Applications (2019)3:37

; https://doi.org/10.1038/s41699-019-0120-8

\section{INTRODUCTION}

The possibility of reaching real-time molecular imaging for cancer diagnosis using time-of-flight positron emission tomography scanners (TOF-PET) calls for well dedicated efforts along this line of research. ${ }^{1}$

Benefits would extend beyond a 10-fold sensitivity, and spread to areas like neonatal imaging and theranostics, just to mention a few. ${ }^{2}$ In terms of detector performance, this translates to reaching a time resolution of $10 \mathrm{ps}$, needed for $511 \mathrm{keV}$ gammas to travel few millimeters within a cancer tumor. At present, the forefront time resolution values obtained in a commercially available TOFPET scanner are at the level of 215 ps using $\mathrm{Lu}_{2-x} \mathrm{Y}_{x} \mathrm{SiO}_{5}$ crystals and silicon photomultiplier (SiPM) readout. ${ }^{3}$ Time resolution achieved with current state-of-the-art scintillators featuring standard photon emission mechanisms, i.e., minimum decay times of tens of nanoseconds and maximum light yield of $100,000 \mathrm{ph} / \mathrm{MeV}$, is largely limited by their associated photon-time density. ${ }^{4-6}$ Hence, harvesting prompt photons from quantum confined direct band-gap semiconductors would highly impact the time resolution of state-of-the-art scintillating detectors. ${ }^{4}$

Recent advances in the readout electronics of coincidence time resolution (CTR) measurements using $511 \mathrm{keV}$ gammas have proven to be able to benefit from prompt emission in the form of Cherenkov photons ${ }^{7}$ for the purpose of improving the time resolution with standard scintillators. Unfortunately, classic Cherenkov emission in high-Z scintillators is limited up to 20 photons emitted per $511 \mathrm{keV}$ gamma interaction., 8 Imposing severe limits in the number of fast photons available in the first $10 \mathrm{ps}$ after the gamma has interacted. Normally, for standard state-of-the-art materials, photon-time density fluctuates $\sim 1$ photon/MeV per picosecond just from the scintillation mechanisms, not considering Cherenkov ${ }^{10}$ or hot intraband luminescence (IBL).$^{11}$ In order to increase the prompt photon emission yield, we propose to replace the usual bulk scintillator pixel by a sampling configuration, so that the recoil electron from a photoelectric conversion created in the dense host scintillator can eventually reach a fast emitting nano-scintillator layer. ${ }^{12}$ In this way, prompt or relatively prompt emission from nanocrystals could be added to the standard scintillating signal and help to decrease the overall timing jitter associated to particle detection. ${ }^{4}$ In this approach, each component brings its own functionality, the fast material is driving the timing improvements and the heavy/standard scintillator provides the stopping power and energy resolution.

One of the first direct band-gap semiconductor nanocrystals studied for the purpose of ultrafast timing detector applications at the level of $10 \mathrm{ps}$ has been CdSe nanoplatelets. ${ }^{13} \mathrm{CdSe}$ with a 1D quantum confinement and a thickness of $\sim 1.5 \mathrm{~nm}$, corresponding to 4-5 monolayers $(\mathrm{ML})^{14}$ has been reported as one of the materials with fastest radiative recombination times due to its giant oscillator strength, ${ }^{15,16}$ a low stimulated emission threshold of $6 \mu \mathrm{J} / \mathrm{cm}^{2}$ and a high biexciton binding energy of $\sim 30 \mathrm{meV}$ at room temperature. ${ }^{17,18}$ Their red-shifted radioluminescence (RL) signal with $25 \%$ of the fast emission being unresolved by a setup with an instrumental response function (IRF) of $\sim 130 \mathrm{ps}$ full width half maximum (FWHM) and the rest being $\sim 300$ ps, makes them potential candidates for a new generation of scintillators. ${ }^{13,19}$ However, their implementation as radiation detectors is technologically challenging owing to intrinsic short-comings such as rather low stopping power of individual nanoparticles, ${ }^{20-23}$ high self-absorption owing to small Stokes-shift of emission ${ }^{24-26}$ and the necessary presence of organic ligands needed for surface passivation and shaping the luminescence and spatial distribution of nanoplatelets. ${ }^{27-31}$

In this contribution, we focus on understanding and demonstrating the timing potential capabilities of these types of materials under ionizing radiation in section 2.1 and 2.2. The

\footnotetext{
${ }^{1}$ CERN, Esp. de Particules 1, Meyrin 1217, Switzerland. ${ }^{2}$ Università degli Studi Milano-Bicocca, Piazza dell' Ateneo Nuovo 1, Milano 20126 , Italy. ${ }^{3}$ Institute of Physics, University of Tartu, W. Ostwald Str.1, Tartu 50411, Estonia. ${ }^{4}$ Universitè de Lyon, Universitè Claude Bernard, Lyon 1, CNRS, Institut Lumière Matière UMR5306, F-69622 Villeurbanne, France. ${ }^{5}$ Department of Chemistry, Ghent University, Krijgslaan 281-S3, 9000 Ghent, Belgium. ${ }^{6}$ Skobeltsyn Institute of Nuclear Physics, Lomonosov Moscow State University, Leninskie Gory, 1(2), 119991 Moscow, Russia. *email: rosana.martinez.turtos@cern.ch
} 
section 2.3 has the purpose of showing how $\mathrm{CdSe} / \mathrm{CdS}$ nanoplatelets could be used in a real detector geometry for medical applications and what are the scintillating features of this type of sample. In the Discussion section, we include a study of the minimum performance needed from the CdSe-based built-up layers to reach the $10 \mathrm{ps}$ goal. CdSe/CdS core-crown nanoplatelets will be studied in combination with state-of-the-art bulk materials such as LYSO, forming drop-casted films or after an embedding procedure that uses polystyrene (PS) as a host matrix. Our aim is to prove that $\mathrm{CdSe}$ is indeed a potential candidate for ultrafast timing applications in combination with standard scintillating technology, specially for TOF techniques using $511 \mathrm{keV}$. A second intention is to delineate a road map for the future application of CdSe nanoplatelets or similar colloidal nanocrystals with sub-1 ns decay times in a hybrid detector pixel geometry, where they are mechanically and chemically stabilized. ${ }^{4,13}$

In this paper, we start from the luminescence properties of individual $\mathrm{CdSe} / \mathrm{CdS}$ nanoplatelets under laser and X-ray excitation and show red-shifted biexcitonic RL at room temperature. We follow-up with a study of the light emission features of a more complex system, in which nanocrystals are either drop-casted on LYSO or forming a nanocomposite. The drop-casting technique using LYSO as direct substrate has been chosen to understand and demonstrate the timing capabilities potential of $\mathrm{CdSe}$ under $511 \mathrm{keV}$ gamma excitation, as this method is capable of using the standard scintillator as a waveguide, transporting light to the photodetector. However, this compromises the performance of the heavy scintillator needed for the detector's energy resolution, so we follow-up with a nanocomposite approach, where CdSe/CdS nanoplatelets are mechanically stabilized on a host matrix, as this is key for real detector applications. Time-resolved ultrafast decay kinetics of a first generation CdSe-based nanocomposite under 100 -fs X-ray excitation is shown together with first light output measurements obtained by X-ray attenuation and cross-checked by spectrally-resolved pulsed cathodoluminescence. We present first time results on the characterization of a sampling scintillating pixel, where standard LYSO $200 \mu \mathrm{m}$-thick plates are combined with $\mathrm{CdSe} / \mathrm{CdS}$ nanoplatelets for $511 \mathrm{keV}$ gamma detection. The promising results on the decay kinetics and time resolution obtained with a still non-optimized CdSe-LYSO sampling pixel are pushing for developing reproducible and scalable methods to produce $\mathrm{CdSe-based} \mathrm{built-up} \mathrm{transparent} \mathrm{nanocomposite} \mathrm{layers} \mathrm{at}$ least $100 \mu \mathrm{m}$ thick in order to substitute the drop-casted films in the hybrid pixel geometry. Drop-casted films can be considered as a random distribution of "unconnected"-emitting centers, in the sense of overall energy distribution, in a given volume and they impede the application of a readout method that guarantees the ultimate performance in terms of combined energy and time resolution previously demonstrated in ref. ${ }^{12}$

\section{RESULTS}

Light emission properties of CdSe/CdS drop-casted films on LYSO The core-crown heterostructure of CdSe-based nanoplatelets has been chosen owing to its enhanced quantum yield and stability compared with bare platelets. ${ }^{32}$ A first characterization of the $\mathrm{CdSe} / \mathrm{CdS}$ luminescent properties under laser and X-ray excitation is performed with highly diluted films in order to avoid selfabsorption. The results are shown in Fig. 1, where a clear redshifted (lower energies) emission can be seen next to the photoluminescence $(\mathrm{PL})$ line. The fit is done using a Voigt probability density function for each spectral line with similar width but centered toward lower energies. The RL data in Fig. $1 \mathrm{~b}$. confirm the presence of a second spectral line red-shifted from $2.385 \mathrm{eV}$ to $2.35 \mathrm{eV}$, i.e., equivalent to a binding energy of $35 \mathrm{meV}$, with a yield fraction of $\sim 0.3$. The result of $35 \mathrm{meV}$ is well in correspondence with the value reported as the binding energy of biexcitons, $\sim 30$ meV for bare CdSe nanoplatelets ${ }^{17}$ and it could indicate the presence of other types of multiexcitonic states. The transmission electron microscope image of the $\mathrm{CdSe} / \mathrm{CdS}$ corecrown nanoplatelets used in this study is shown in Fig. 1a together with the PL emission spectra. The effective decay time under nonintense laser excitation has been measured to be $\sim 600 \mathrm{ps,} \mathrm{which} \mathrm{is}$ in very good agreement with the $520 \mathrm{ps}$ found for CdSe nanoplatelets without crown. ${ }^{13}$ Under X-ray excitation, we obtain a faster emission towards the higher wavelengths, which reassures that the red-shifted emission is not a self-absorption artifact. The effective RL decay time is $\sim 160 \mathrm{ps}$, which corresponds to both excitonic and biexcitonic spectral lines, yielding a PL/RL factor of 3.75. For further information regarding biexcitonic emission under pulsed laser excitation at different laser powers refer to Section S1.

For the purpose of detecting highly energetic particles, CdSe nanoplatelets are studied in combination with high-Z materials able to perform as state-of-the-art scintillators. The a)
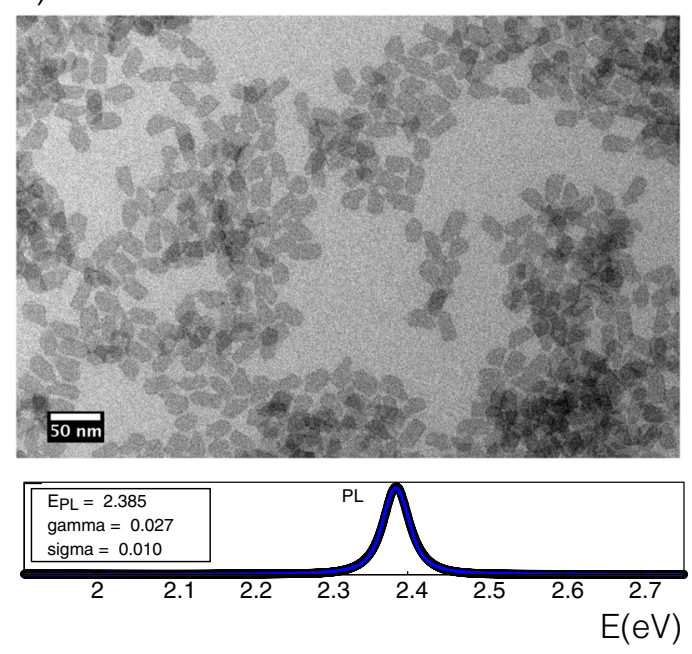

b)

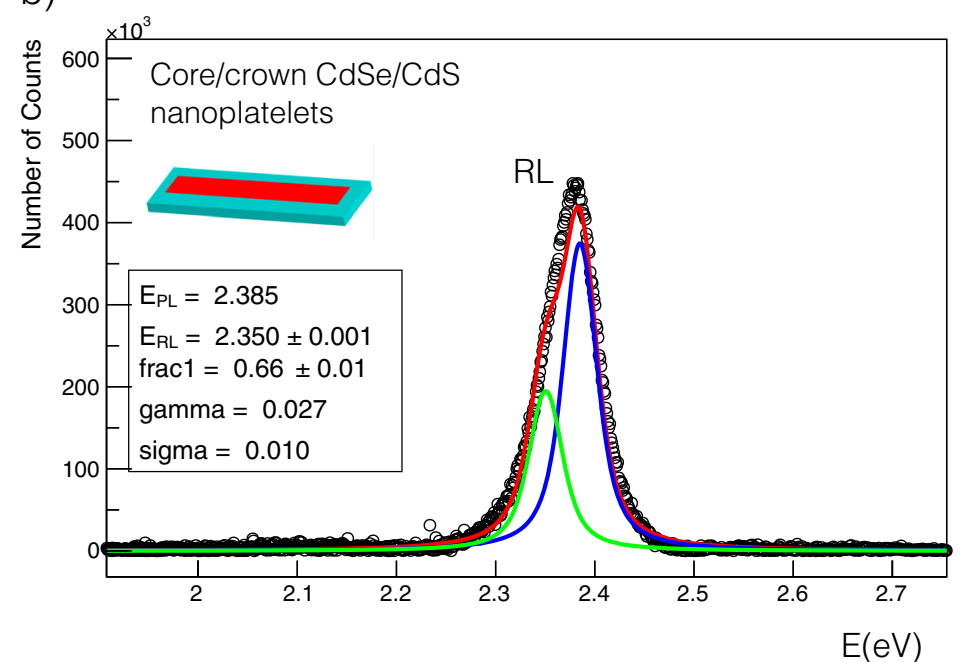

Fig. 1 Photoluminescence (PL) and radioluminescence (RL) energy spectra of core-crown CdSe/CdS nanoplatelets highly diluted films. a The TEM image for the CdSe/CdS synthesis is included above the PL spectra. The scale bar indicates the length of $50 \mathrm{~nm}$. $\mathbf{b}$ The RL data confirm the presence of a second spectral line red-shifted from $2.385 \mathrm{eV}$ to $2.35 \mathrm{eV}$, i.e., equivalent to a binding energy of $35 \mathrm{meV}$, with a yield fraction of $\sim 0.3$. The Voigt function parameters characteristic of the PL spectra are shown separately in plot a) 


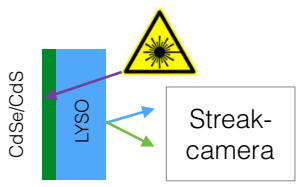

a)

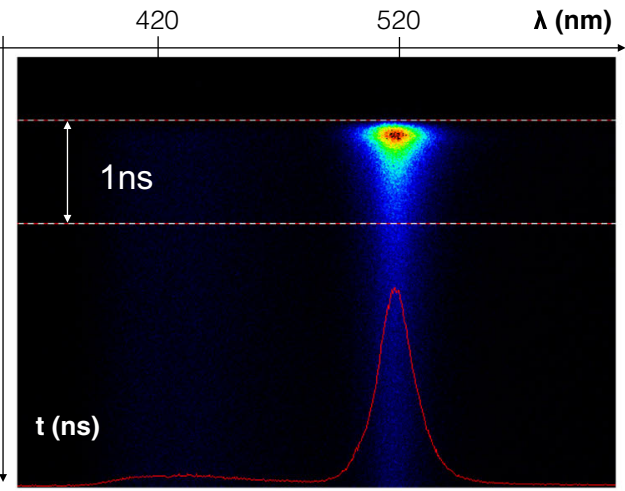

c)

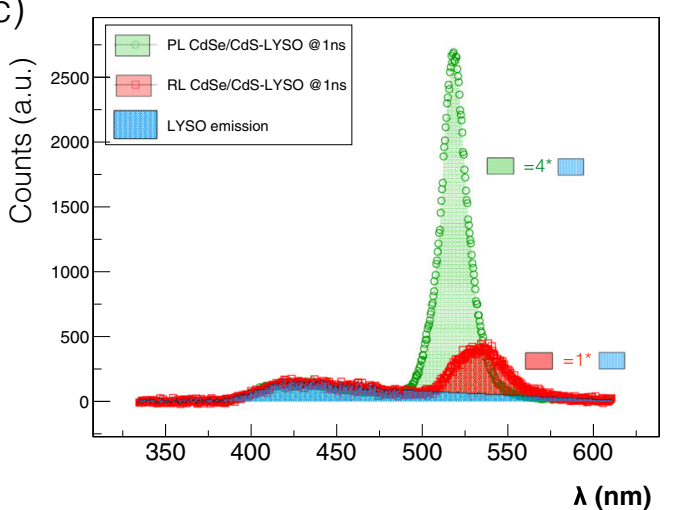

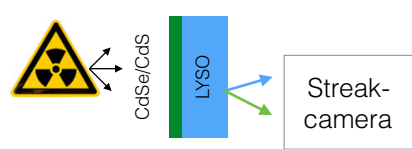

b)

420 520 $\lambda(\mathrm{nm})$

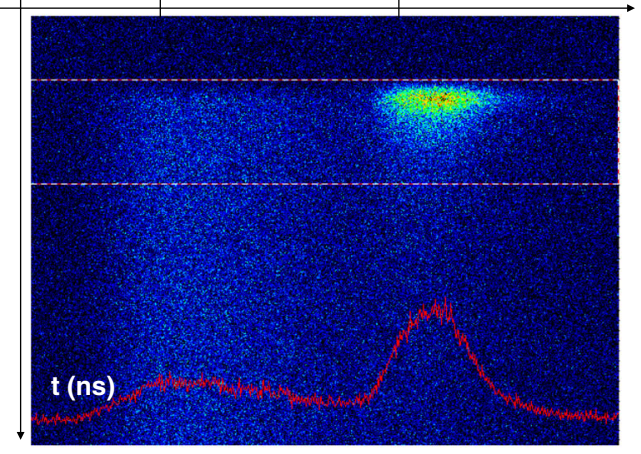

d)

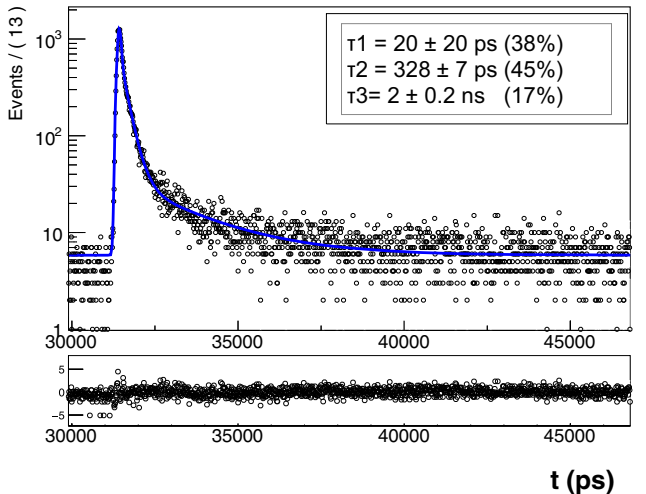

Fig. 2 Spectral-time-resolved characterization of $\mathrm{CdSe} / \mathrm{CdS}$ core-crown nanoplatelets drop-casted on LYSO. Photoluminescence a and radioluminescence $\mathbf{b}$ time-resolved spectra of a CdSe/CdS drop-casted film deposited on a LYSO:Ce substrate as readout by a Hamamatsu streak camera. c Comparison of PL and RL CdSe/CdS drop-casted film spectra integrated over the first nanosecond normalized to LYSO emission. $\mathbf{d}$ Time-resolved $\mathrm{CdSe} / \mathrm{CdS}$ core-crown radioluminescence integrated from $200-800 \mathrm{~nm}$ as measured with a hybrid PMT in time correlated single photon counting (TCSPC) mode with 130 ps FWHM instrumental response function

spectral-time-resolved PL and RL obtained for the $\mathrm{CdSe} / \mathrm{CdS}$ drop-casted film on LYSO is shown in Fig. 2. The excitation and corresponding readout geometry for each measurement is shown in the schematics above the streak camera figures.

As expected, the PL emission spectra of core-crown CdSe/CdS nanoplatelets (NPLs) is centered at $515 \mathrm{~nm}$ and in comparison with the LYSO PL emission integrated over the first nanosecond, the signal is at least four times higher for CdSe. Taking into account the LYSO emission in the first nanosecond (1000 ph/ $\mathrm{MeV}^{33}$ ) and the characteristics decay times exhibited by CdSe $\left(300 \mathrm{ps}^{13}\right)$, this measurement points towards a CdSe/CdS photontime density at least 10 times higher than LYSO. In contrast to the $\mathrm{PL}$ results, under $\mathrm{X}$-ray excitation we observe a large red-shift in the RL emission centered at $\sim 530 \mathrm{~nm}$, together with a decrease in the emission intensity of around a factor $75 \%$ as shown in Fig. 2c. These highlights the self-absorption issues of the drop-casted films. The comparison between $\mathrm{PL}$ and $\mathrm{RL}$ was done without moving the sample in order to keep constant the uncertainties related to focusing and alignment.

Figure $2 \mathrm{~d}$ shows the decay kinetics of $\mathrm{CdSe} / \mathrm{CdS}$ core-crown nanoplatelets under X-ray excitation. In this case, the film was drop-casted on glass to avoid the influence of LYSO emission and excited with a pulsed X-ray tube with a FWHM of $\sim 50 \mathrm{ps}$. The photodetector used for the readout is a fast hybrid PMT and the instrumental response (IRF) function of the whole setup has been obtained measuring the IBL of $\mathrm{Li}_{2} \mathrm{MoO}_{4}{ }^{11}$ The IRF has a full width half maximum of $\sim 130 \mathrm{ps}^{34}$ and this is the reason for the unresolved first decay component expressed as $20 \pm 20$ ps.

CdSe-LYSO sampling pixel under $511 \mathrm{keV}$ excitation

One of the benefits of improving the time resolution of scintillating detectors is applied in the TOF technique. TOF is used for particle identification in high-energy physics experiments or as a way to spatially resolve the point of positron annihilation in PET scanners. For the latest, $511 \mathrm{keV}$ gammas are detected in coincidence usually by means of LYSO crystals and SiPM readout. In this section, we combine several LYSO $3 \times 3 \times 0.2 \mathrm{~mm}^{3}$ plates coated with the $\mathrm{CdSe} / \mathrm{CdS}$ drop-casted film and we proceed with the assembly of 10 plates to form a $3.8 \times 3.8 \times 3 \mathrm{~mm}^{3}$ pixel shown in Fig. 3a. The aim for this kind of construction is to stop $511 \mathrm{keV}$ gammas in the LYSO plates and excite the CdSe/CdS drop-casted films with the recoil electron created upon the photoelectric effect. Owing to the characteristic travel path of the recoil electron with $>400 \mathrm{keV}$, a fraction of them will be able to leak some of their energy to the nano-material and this can be observed by measuring the decay kinetics of the sampling pixel.

The time-resolved photon emission of the CdSe-based sampling pixel consisting of 10 LYSO plates $200 \mu \mathrm{m}$ thick covered by the $\mathrm{CdSe} / \mathrm{CdS}$ drop-casted films is shown in Fig. 3b. In this case, the readout takes into account all events no matter the energy 
a)

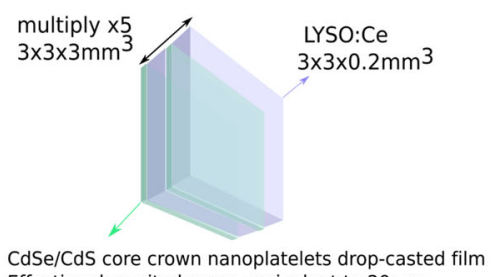

Effective deposited mass equivalent to $20 \mu \mathrm{m}$

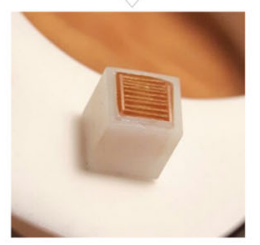

C)

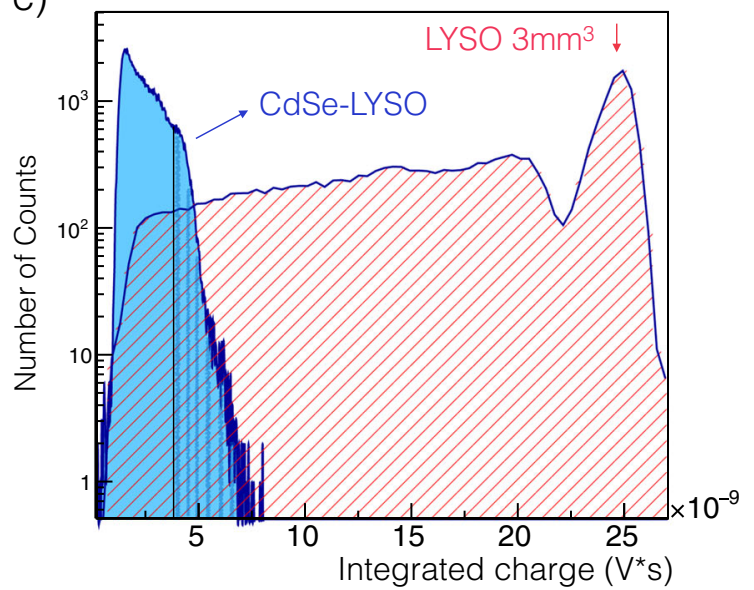

b)
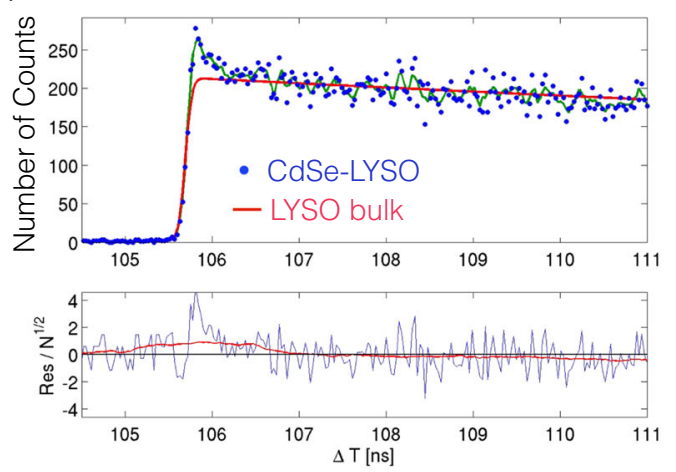

d)

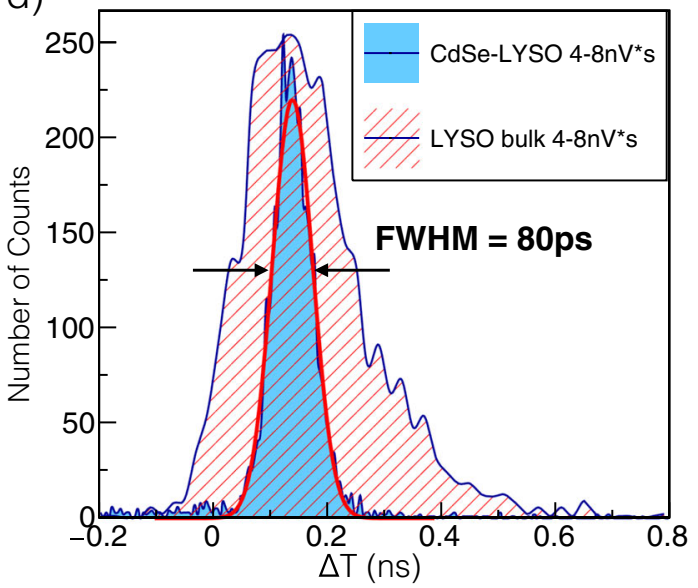

Fig. 3 Scintillation properties of the CdSe-LYSO sampling pixel measured under $511 \mathrm{keV}$ gamma excitation. a Schematics and photo of the CdSe-LYSO sampling pixel. b Rise time measured in a time correlated single photon counting setup with IRF of 150 ps. ${ }^{4}$ c Energy spectrum obtained with a Na ${ }^{22}$ source, coupling the CdSe-LYSO sampling pixel to an FBK NUV-HD SiPM and triggering in coincidence with a LSO:Ce:Ca co-doped reference crystal. The same spectrum taken with a bulk $3 \times 3 \times 3 \mathrm{~mm}^{3}$ LYSO crystals is shown for comparison. d Delay time distribution for all CdSe-LYSO events in coincidence with integrated charge higher that $4 \mathrm{nVs}$. The time distribution of LYSO bulk events having a similar integrated charge between 4 and $8 \mathrm{nVs}$ is shown as a reference

deposited, therefore the signal shown is characteristic for the average type of event. We observe a very fast rise time, characteristic of the first rise time component of LYSO:Ce crystals ${ }^{35}$ and a prompt peak on top of the LYSO emission. The red line follows the rise time parameter of $\sim 10 \mathrm{ps}$ and the green curve represents 3-4 bins average. This measurement constitutes an experimental proof that $511 \mathrm{keV}$ gamma excitation is able to transmit part of its energy to a built-up nano-scintillating layer in direct contact with the LYSO plates.

The $511 \mathrm{keV}$ energy spectrum obtained by integrating the measured charge when coupling the CdSe-LYSO sampling pixel to a FBK NUV-HD SiPM ${ }^{36}$ is shown in Fig. 3c. The events are in coincidence with a LSO:Ce:Ca $2 \times 2 \times 3 \mathrm{~mm}^{3}$ co-doped crystal used as reference with a coincidence time resolution (CTR) of $60 \mathrm{ps}$ FWHM. ${ }^{7}$ As a form of direct comparison, the integrated charge obtained when measuring a bulk $3 \times 3 \times 3 \mathrm{~mm}^{3}$ LYSO:Ce crystal from the same producer than the coated plates is shown together with the CdSe-LYSO data.

As expected from the optical properties of $\mathrm{CdSe} / \mathrm{CdS}$ nanoplatelets, their direct drop-casting on the surface of the LYSO disrupts the angle of total internal reflection and therefore deteriorates the index-light-guiding to the point that the photopeak and the Compton edge are not longer well separated as for the case of bulk LYSO (red stripes spectrum).

According to Fig. 3c, there is a light output reduction for the CdSe-LYSO sampling pixel of at least a factor 5 , not taking into account the SiPM saturation issues. In terms of CTR, which scales down as the square root of the photon-time density, a deterioration of a factor 5 in the number of photoelectrons detected would imply a worsening of the pixel timing performance of about a factor 2.2. Assuming plain LYSO emission, this factor translates to an expected time resolution of $\sim 180$ ps FWHM. However, looking at the delay time distribution of events in the CdSe-LYSO sampling pixel with an integrated charge higher that $4 \mathrm{nV}^{*} \mathrm{~s}$, we obtain a CTR value of $80 \mathrm{ps}$ FWHM. For comparison, we have included in Fig. $3 d$ the delay time distribution obtained with bulk LYSO crystal, choosing events with a similar integrated charge from 4 to $8 \mathrm{nV}^{*} \mathrm{~s}$.

Despite all the optical issues, drop-casted films allow to mechanically assemble a CdSe-LYSO sampling pixel for a first time proof-of-concept with $511 \mathrm{keV}$ gammas. An extended study of a second type of sampling pixel using $\mathrm{Bi}_{4} \mathrm{Ge}_{3} 3 \mathrm{O}_{12}$ as high-Z material is included in the Supplementary Information Section S2, Fig. S2. The CTR of this crystal is determined by its Cherenkov emission at a level of few photons, instead of by its standard photostatistic and this has a crucial role in the readout threshold and depth of interaction correction needed in long sensors. Therefore, the study of this type of combination, Cherenkov emitter and fast nanocrystals is complementary to the LYSO case.

The results presented above constitute an experimental proof that CdSe nanoplatelets bears significant timing potential for $511 \mathrm{keV}$ gamma detection. However, its assembly in combination 

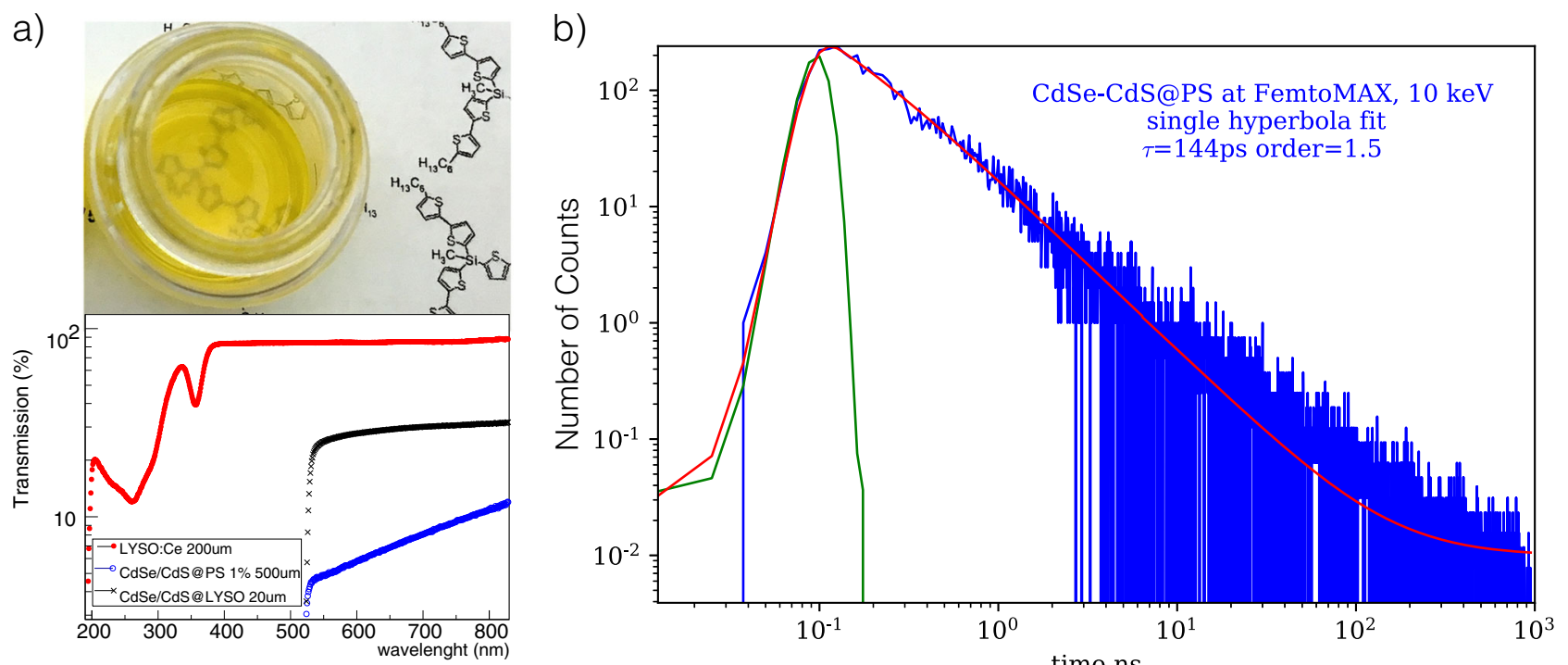

Fig. 4 Scintillating kinetics of CdSe-based nanocomposite $1 \%$ weight concentration. a Transmission of CdSe/CdS nanocomposite $1 \%$ weight concentration $500 \mu \mathrm{m}$ thick and CdSe/CdS drop-casted film with an effective thickness of $20 \mu \mathrm{m}$ in comparison with LYSO:Ce crystal $200 \mu \mathrm{m}$ thick. b Time-resolved decay kinetics of CdSe/CdS@PS 1\% as measured at the FemtoMAX beamline with a IRF of 38 ps FWHM. The doublelogarithmic scale has been chosen for better visualization of the data

with state-of-the-art scintillators must be optimized. This work proves that CdSe nanoplatelets, if properly stabilized as emitting centers in a matrix host, would revolutionize the field of fast timing research and the new detectors could eventually reach the regime of real-time molecular imaging in the clinical scenario. Our efforts in this direction are described in the section below.

Light emission properties of CdSe-based nanocomposite under Xray and electron excitation

The decay kinetics of $\mathrm{CdSe} / \mathrm{CdS}$ nanocomposite in a polystyrene (PS) host (CdSe/CdS@PS) with 1\% weight concentration has been obtained with exceptional time resolution of 38 ps FWHM at the FemtoMAX beamline in Lund, Sweden. Time-resolved data are shown in Fig. 4 together with the setup IRF. We observe a fast photo-response, which decays two order of magnitudes in the first 10 ns according to Fig. 4b, (for further fitting details with multiexponential decay components refer to Table S1). These results are found to be in good agreement with the PL study performed with similar nanoplatelets using long integration gates up to $20 \mathrm{\mu s}^{32}$ In addition to the rise-decay kinetics, Fig. 4a shows the prepared sample and its transmission in comparison with $\mathrm{CdSe} /$ CdS drop-casted films and LYSO plates up to $200 \mu \mathrm{m}$ thick.

Concerning light output under high excitation, the two-step light yield characterization results obtained for the CdSe-based nanocomposite sample are shown in Fig. 5. Both samples, LYSO and CdSe/CdS@PS 1\% have the same cross section size of $3 \times$ $3 \mathrm{~mm}^{2}$ and were excited by a collimated X-ray beam of $3 \mathrm{~mm}$ diameter. The CdSe/CdS@PS 1\% light output is obtained by comparing to the amount of photons integrated over a predefined $500 \mathrm{~ns}$ gate using the LYSO as reference. Taking into account LYSO intrinsic yield ${ }^{33}$ and its reported non-proportionality of $\sim 55 \%$ for $10 \mathrm{keV}$ gammas, we obtain a reference value of $22,000 \mathrm{ph} / \mathrm{MeV}$. A direct comparison of the decay curves obtained for both samples, LYSO (reference) and CdSe/CdS@PS 1\% is shown in Fig. $5 \mathrm{a}$ for the first $10 \mathrm{~ns}$ of scintillating signal next to the sample $X$-ray attenuation. For further details regarding the light yield determination measurements consult the Methods section and for a full review using different materials refer to ref. ${ }^{34}$

A summary of the measured absorption coefficient and comparative light yield value obtained for the CdSe-based nanocomposite sample is shown in Table 1. Summing up the two-step light yield measurements result in a value no higher than $100 \mathrm{ph} / \mathrm{MeV}$.

A second method to determine the light yield of the nanocomposite sample uses short range electron excitation. The procedure of pulsed cathodoluminescence yield comparison of transparent samples (crystals or PS slabs) was described in. ${ }^{37}$ Similarly to the drop-casted $\mathrm{CdSe} / \mathrm{CdS}$ nanoplatelets under continuous electron beam, ${ }^{34}$ the same nanoplatelets embedded in polystyrene (PS) have shown some degree of $\mathrm{CL}$ degradation under $120 \mathrm{keV}$ electron pulses. The peak pulse electron current was reduced to $0.7 \mathrm{~A} / \mathrm{cm}^{2}$ to partially mitigate this effect. Owing to the pulsed nature of the source, the first spectrum was recorded with no preliminary irradiation and can be considered unaffected by degradation as shown in Fig. 6, curve 1. The repeated measurement indicated $\sim 20 \%$ loss of yield after full spectral scan (which takes $\sim 300-400$ electron pulses).

Figure 6 shows the spectra of CdSe/CdS@PS and LYSO:Ce, recorded in 0-200 $\mu$ s time window in the same conditions. Such time window was selected to ensure that all the luminescence has completely decayed during acquisition, so that the total yield of different emissions can be compared. The spectrum consists of three separate emission bands: PS host luminescence at $330 \mathrm{~nm}$, $\mathrm{CdSe} / \mathrm{CdS}$ excitonic band at $525 \mathrm{~nm}$ and broad defect emission at $\sim 750 \mathrm{~nm}$. The absence of the latter in the spectrum recorded in 0-32 ns time window indicates that it has the decay constant in microsecond range, which could not be measured in our experiment. The decay curves of $330 \mathrm{~nm}$ and $525 \mathrm{~nm}$ bands are complex and the list of their exponential decay components is shown in Table S1. Both high-energy X-rays and electron beam pulses populate high excited states, wherefrom energy is transferred to the emission centers through various relaxation processes causing appearance of complicated decay curves. It should be noted that the main component of PS emission (15 ns) is very similar to the slow component of excitonic emission (18 ns), which indicates that about half of the total excitonic yield in cathodoluminescence experiment comes from re-absorption of slow PS emission. Comparing decay kinetics between the different experiments shows that the decay component of $300 \mathrm{ps}$ is only preserved for the X-ray experiment at CERN where the beam flux does not degrade the luminescence properties of $\mathrm{CdSe} / \mathrm{CdSe} @ \mathrm{PS}$ $1 \%$ sample. For an extended study of this method with a different 

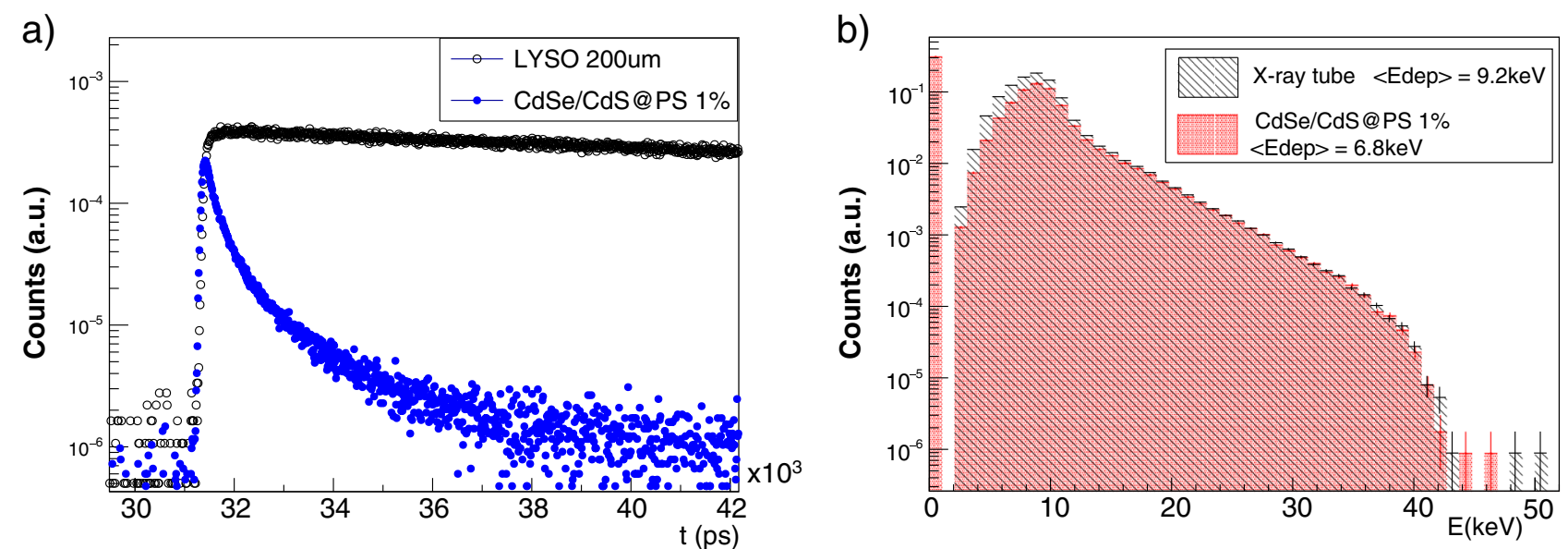

Fig. 5 Two-step light yield determination of CdSe/CdS@PS 1\% sample. a Comparative light output between LYSO and CdSe/CdS@PS 1\% obtained in a time correlated single photon counting setup with a tungsten pulsed X-ray tube up to $40 \mathrm{kV}$. Data are shown within a 10 ns gate for visual purposes. b X-ray attenuation measured with the Timepix chip detector ${ }^{45}$

Table 1. Measured absorption coefficient $\eta$, comparative light output and light yield for a CdSe/CdS@PS 1\% nanocomposite sample measured under soft X-ray excitation up to $40 \mathrm{keV}$

\begin{tabular}{|c|c|c|c|c|c|}
\hline Nano-scint & $L Y_{\text {est }}^{1} \%$ & $\eta_{T P X}$ & $L Y_{\text {meas }}(p h / M e V)$ & Yield $_{1 n s}^{2} \%$ & Yield ${ }_{10 n s}^{2} \%$ \\
\hline CdSe/CdS@PS 1\% & 0.1 & 0.26 & 85 & 60 & 83 \\
\hline \multicolumn{6}{|c|}{$\begin{array}{l}{ }^{1} \text { Estimated light yield as compared with the LYSO intrinsic light yield at } \\
10 \mathrm{keV} \text { and assuming full energy deposition } \\
{ }^{2} \text { The Yield1ns and Yield } 10 \mathrm{~ns} \text { represent the percentage of the emission } \\
\text { integrated over } 1 \mathrm{~ns} \text { or } 10 \mathrm{~ns} \text { with respect to the total emission integrated } \\
\text { over } 500 \mathrm{~ns}\end{array}$} \\
\hline
\end{tabular}

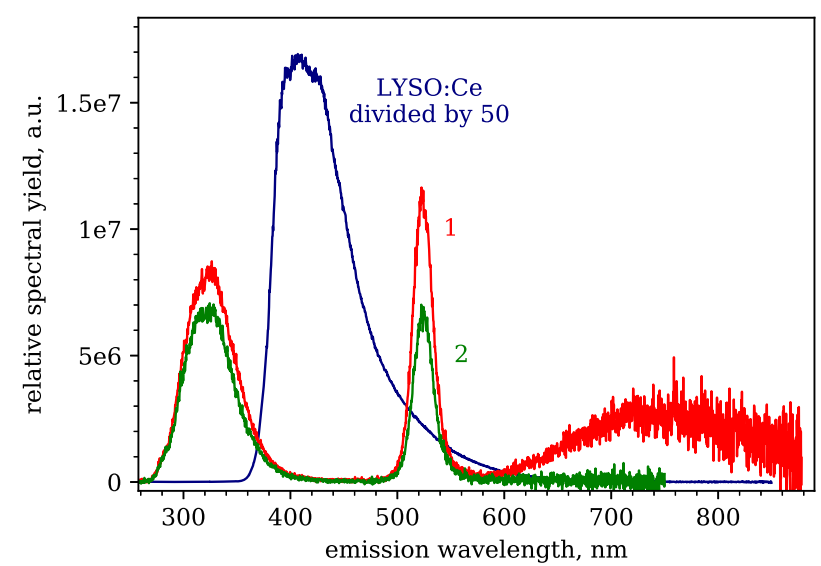

Fig. 6 High-energy cathodoluminescence emission spectra of CdSe/ CdS@PS 1\% Curve (1) was recorded in the $0-200 \mu$ s time gate and curve (2) between 0 and 32 ns time window relative to the excitation pulse. The CL spectrum of LYSO:Ce was recorded in $0-200 \mu \mathrm{s}$ time window

type of nanocrystals refer to the Supplementary information Section S4.

The approximate light yield numbers can be derived from the first spectra for which the degradation of the sample was negligible (Fig. 6, curve 1). The total light yield of $525 \mathrm{~nm}$ excitonic band in $0-200 \mu$ s time window is $0.37 \%$ of total LYSO:Ce cathodoluminescence yield. The non-proportionality of LYSO:Ce at $100-120 \mathrm{keV} \mathrm{X}$-ray is $80-85 \%$ relative to the yield at $662 \mathrm{keV} .^{38}$ Assuming the same non-proportionality factor for the electron beam, the excitonic emission yield ( $525 \mathrm{~nm}$ line) is $122 \mathrm{ph} / \mathrm{MeV}$, of which only $68 \mathrm{ph} / \mathrm{MeV}$ belong to the ultrafast components with decay constant $\tau \leq 1.1 \mathrm{~ns}$. For comparison, the yield of PS emission band and defect band is $0.63 \%$ and $0.62 \%$ of LYSO:Ce, respectively, and, combined together, comprise $77 \%$ of total light output of CdSe/CdS@PS.

In this experiment, we can assume that most of the energy is absorbed by PS because the electron penetration depth is no more than $0.2 \mathrm{~mm}$ and most of the material encountered by electrons on that distance is PS. Therefore, the ratio of excitonic to PS emission in this case will correspond to the energy transfer efficiency from PS to NPLs. The ratio of surfaces under $520 \mathrm{~nm}$ and $320 \mathrm{~nm}$ bands in Fig. 6 , curve 1 is 0.58 . However, not all of the $520 \mathrm{~nm}$ emission is caused by prompt processes, and to estimate the efficiency of prompt energy transfer we have to subtract the effect of delayed energy transfer. The total yield of PS and excitonic bands is 0.63 and $0.37 \%$ of LYSO standard, respectively. In all, $55 \%$ of excitonic emission is prompt ( $\tau \leq 1.1 \mathrm{~ns})$, and the rest is somehow delayed. Owing to the similarity of decay components of delayed NPLs emission and PS emission (as described above) we can assume the main reason of delayed energy transfer is re-absorption. Taking the quantum efficiency of NPLs as $\sim 50 \%$, ${ }^{32}$ we can estimate which portion of PS emission was reabsorbed by NPLs. After correcting for re-absorption in this way, the ratio of intrinsic PS yield to prompt excitonic yield becomes 0.21 . This value is in a very good correspondence with $20-25 \%$ obtained by theoretical considerations (Supplementary information Section S3.).

\section{DISCUSSION}

As presented in the sections above, $\mathrm{CdSe} / \mathrm{CdS}$ nanoplatelets exhibit a red-shifted RL emission with a binding energy of $35 \mathrm{meV}$, characteristic of biexcitons in $1.5 \mathrm{~nm}$ thick CdSe platelets. This confirms biexcitonic emission under X-ray excitation, which have associated decay times of $\sim 100 \mathrm{ps}$, a promising route to pursue for fast timing applications.

As expected, CdSe/CdS drop-casted films using LYSO as substrate affect the light transport within LYSO itself, given by the angle of total internal reflection. Optically separating the standard and the fast photon emission phases would allow for a better performance of the sampling pixel. In this regard, we studied the luminescence properties of $\mathrm{CdSe} / \mathrm{CdS}$ nanoplatelets embedded in PS as a way to build a nanocomposite suitable for 
LYSO $100 \mu \mathrm{m}+$ CdSe@PS 60\% 100 $\mu \mathrm{m}$

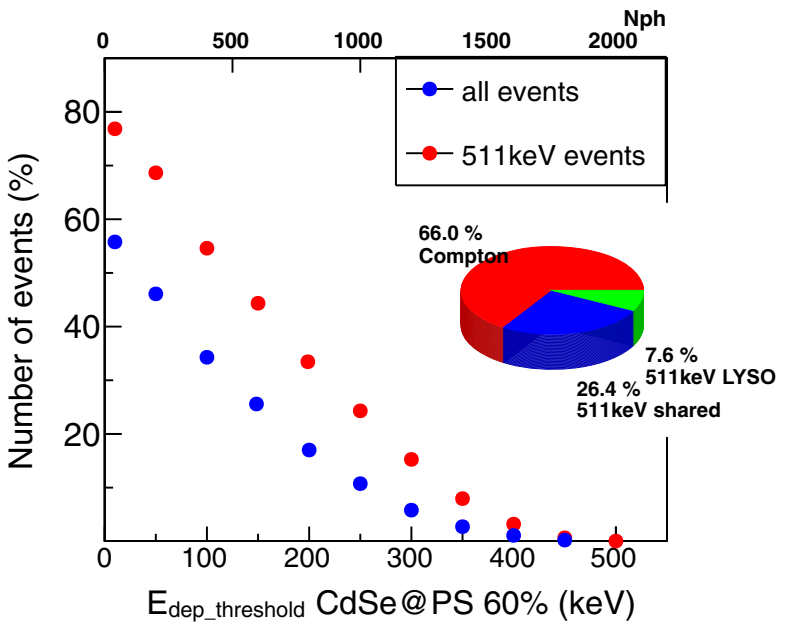

Fig. 7 In total, $511 \mathrm{keV}$ gamma energy distribution in a sampling pixel geometry able to provide ultimate timing. Geant4-based simulations were done for a hybrid pixel consisting of $100 \mu \mathrm{m}$ thick LYSO plates combined with $100 \mu \mathrm{m}$ thick layers of CdSe-based nanocomposite with a loading fraction of $60 \%$ in polystyrene. The blue (red) solid dots show the \% of events with respect to the total number of detected events ( $511 \mathrm{keV}$ events) for which the energy deposited in the nano-phase surpass the energy value indicated in the $x$ axis. The number of photons given per energy deposited in the upper $X$ axis corresponds to the minimum estimated value able to provide ultimate time resolution at energies above $250 \mathrm{keV}$ (see Fig. S6)

the detection of high energetic particles in combination with high-Z materials. The light output of this nanocomposite with $1 \%$ weight concentration have been characterized using X-ray and electron excitation, yielding very modest values of $\sim 100 \mathrm{ph} / \mathrm{MeV}$. Calculations of the range for non-radiative energy transfer between the organic host and the inorganic nanoparticles are able to estimate an effective volume surrounding the nanoplatelets for which the transfer is highly probable (see Fig. S4). For the given weight concentration of $1 \%$, effective non-radiative energy transfer accounts at most for just $20 \%$ of the energy deposited in the PS host. Weight concentrations of at least $10 \%$, where the average distance between nanoplatelets would be on the order of $30 \mathrm{~nm}$ would significantly increase the amount of energy transfer by dipole-dipole interactions, i.e., up to $20 \%$ without considering exciton diffusion (see Section S3 of Supplementary information).

A sampling pixel geometry with optically separated scintillators has been previously implemented and proven to bring better timing to a fraction of the $511 \mathrm{keV}$ events. ${ }^{12}$ The experimental proof-of-concept readout has been implemented with BC-422, a plastic scintillator in the shape of $200 \mu \mathrm{m}$ thin plates combined with $200 \mu \mathrm{m}$ thin plates of LYSO or BGO. This plastic scintillator presents a very low density of $1.0 \mathrm{~g} / \mathrm{cm}^{3}$ (no-photopeak), a minimum decay time of $1.3 \mathrm{~ns}$, light yield of $10,000 \mathrm{ph} / \mathrm{MeV}$ and therefore an excellent CTR of 35 ps FWHM. Therefore, substituting the BC-422 material by CdSe-based nanocomposites layers up to $100 \mu \mathrm{m}$ thick with weight concentrations of minimum $10 \%$ and ultimate timing constitutes a solution for the material bottlenecks faced in fast timing applications.

In this regard, the last developments in the production of high-loaded nanocomposites with weight concentrations up to $60 \%$ are showing excellent transmission values at the level of $60-80 \%$ for $2 \mathrm{~mm}$-thick samples as reported in. ${ }^{31}$ In this study, they report on a chemical path that allows for ligandfunctionalized $\mathrm{CdZnS} / \mathrm{CdS}$ quantum dots to be covalently bound to the polymer host, resulting in nanocomposites samples with high light yield and enhanced probability of gamma conversion.
The inclusion of a dye molecule acting as a wavelength shifter enlarges the small Stoke-shift characteristic of direct band-gap semiconductors and modulates the sample's transparency to values up to $80 \%$. In the case of CdSe nanoplatelets, particles synthesized with 5 monolayers (ML) instead of $4 \mathrm{ML}$ (the ones presented in this study) have been proven to emit at $550 \mathrm{~nm}$ and to present ultrafast interplate Förster energy transfer when used in combination with the $4 \mathrm{ML}$ nanopletelets. ${ }^{39}$ This ultrafast energy transfer mechanism with lifetimes of $\sim 28$ ps between emitting centers with an spectral overlap could be used as a non-radiative wavelength shifting process able to preserve fast rise-times critical for timing applications.

Aiming at the optimization of such hybrid pixel, Geant4 simulations ${ }^{40,41}$ of the energy deposited by $511 \mathrm{keV}$ gamma rays are used for the estimation of the fraction of energy going to each scintillator on an event-by-event basis. This distribution is shown in Fig. 7 for a hybrid pixel composed of LYSO plates $100 \mu \mathrm{m}$ thick and CdSe-based nanocomposite with a loading fraction of $60 \%$, also $100 \mu \mathrm{m}$ thick. The total number of events detected are $\sim 20 \%$ of the total, which compares to $22 \%$ for the case of bulk LYSO $3 \times 3 \times 3 \mathrm{~mm}^{3}$. Regarding the distribution of events fully depositing $511 \mathrm{keV}$ within the sampling pixel they account for $34 \%$ of the gammas detected. We observe that a minority of such events, $8 \%$ are not able to share energy and therefore will carry standard timing resolution. However, the majority of $511 \mathrm{keV}$ events, more than two-thirds, would enter in the category of shared events, having energy deposited in both scintillators. For this configuration, they will represent $26 \%$ of the events and they will exhibit improved time resolution.

These calculations show the incredible application potential of having a highly loaded mono-disperse nanocomposite up to $100 \mu \mathrm{m}$ thick in combination with LYSO or other high-Z scintillators with similar density. Not only the total number of detected events is $90 \%$ compared with bulk LYSO, but the number of events depositing $>200 \mathrm{keV}$ in CdSe-based nanocomposite represents $\sim 30 \%$ of the events depositing $511 \mathrm{keV}$ in the sampling pixel. The transmission/transparency of a mono-disperse highly loaded nanocomposite with thickness of $100 \mu \mathrm{m}$ is a topic for further investigation, as well as the chemical path leading to such high loadings for this type of nanoplatelets. ${ }^{31}$

This paper reports on the very encouraging results from a proofof-concept experiment exploring the use of CdSe-based nanoplatelets in combination with standard detector technology in a hybrid functional pixel. Integrating fast emitting nanocrystal semiconductors with state-of-the-art scintillators is shown here as a pathway for improved timing performance, which otherwise faces severe limitations.

We are able to observe for the first time $\mathrm{CdSe} / \mathrm{CdS}$ scintillation emission under $511 \mathrm{keV}$ gamma excitation in the form of a prompt peak added to the LYSO characteristic kinetics. This prompt emission has proven to reduce the timing jitter associated with $511 \mathrm{keV}$ gamma detection, yielding CTR state-of-the-art values of $\sim 80$ ps.

In view of the upgraded detector pixel geometry, we foresee the need for a CdSe-based built-up nano-scintillating layer with a minimum thickness of $100 \mu \mathrm{m}$. Aiming at higher nanocomposite loadings of at least $10 \%$ weight concentration is essential in order to harvest most of the energy deposited in the polymer matrix. On top, increasing the total light yield of CdSe-based nanocomposite in a factor 100, would provide with the photon-time density critical to reach ultimate time resolution for a significant fraction of the events fully contained in the proposed hybrid pixel geometry.

This proof-of concept test opens the way to the design and study of larger size sensors aiming at a high sensitivity and picosecond timing resolution in the low energy detection regime of few hundreds keV. 


\section{METHODS}

Luminescence properties of CdSe/CdS core-crown nanoplatelets CdSe-based samples consist of CdSe $4 \mathrm{ML}$ in the form of nanoplatelets synthesized by wet chemical methods (protocol established in ${ }^{15}$ ). After the main synthesis, a second one follows in order to add a CdS crown around the CdSe nanoplatelets as reported in ref. ${ }^{42}$

The spectroscopy of drop-casted $\mathrm{CdSe} / \mathrm{CdS}$ films using LYSO as substrate was measured with a Hamamatsu streak camera model C10910. The PL was obtained using a PILAS picosecond pulsed diode laser with wavelength of $372 \mathrm{~nm}$ as source of excitation with $\sim 45 \mathrm{ps}$ FWHM. Meanwhile, the RL data use a Hamamatsu 50 ps FWHM pulsed Xray tungsten tube with energies up to $40 \mathrm{keV}$ and lines centered between 9 and $11 \mathrm{keV}$. For these measurements, we have used LYSO:Ce from Crystal Photonics Inc., $3 \times 3 \times 0.2 \mathrm{~mm}^{3}$ as a substrate for the film preparation and the nanoplatelet deposition covers one of the two $3 \times 3 \mathrm{~mm}^{2}$ polished faces. The emission is collected in transmission mode through the $200 \mu \mathrm{m}$ thick LYSO crystal and integrated over the first $5 \mathrm{~ns}$. The effective thickness of the film deposited on LYSO used for the PL and RL measurements is estimated to be $\sim 20 \mu \mathrm{m}$ given by the amount of CdSe deposited and assuming all the nanoplatelets are forming a bulk layer.

\section{CdSe-LYSO sampling pixel under 511 keV gamma excitation}

For the rise-decay time measurement under $511 \mathrm{keV}$ excitation, we use a time correlated single photon counting (TCSPC) setup with an IRF of $150 \mathrm{ps}$ FWHM measured with Cherenkov emission in undoped LuAG crystals. The start detector consist of a LSO:Ce:Ca co-doped crystal coupled to a SiPM and an ID Quantique performs as stop detector. For more details regarding the specifications and previous data taken with this setup please refer to ref. ${ }^{35}$

The CTR measurements were performed in the state-of-the-art experimental setup previously described in. ${ }^{7,12}$ This bench uses a LSO:Ce:Ca $2 \times$ $2 \times 3 \mathrm{~mm}^{3}$ crystal as reference detector with a single time resolution of 41 ps FWHM. The SiPMs in used are FBK NUV-HD with $40 \mu \mathrm{m}$ SPADs size ${ }^{36}$ and measured single photon-time resolution of $70 \mathrm{ps}$. The electronics readout divides the integrated signal to perform separate measurements of energy and timing and a high-frequency amplifier ${ }^{43}$ with $1.5 \mathrm{GHz}$ bandwidth is used to record the time stamps. The signals are analyzed in a Lecroy oscilloscope with $3.5 \mathrm{GHz}$ bandwidth and $40 \mathrm{Gs} / \mathrm{s}$ (using four channels this reduces to $20 \mathrm{Gs} / \mathrm{s}$, i.e., 50 ps binning). The sampling pixels are coupled to the SiPMs using Meltmount glue with an index of refraction of 1.582 , which allows for a good index matching with the photodetector.

$\mathrm{CdSe} / \mathrm{CdS}$ embedded in polystyrene at $1 \%$ weight concentration The CdSe-based nanocomposite followed an embedding procedure where the colloidal nanoplatelet solution is mixed with dissolved pure PS, evaporated and dried. The $\mathrm{CdSe} / \mathrm{CdS}$ core-crown solution has been synthesized with a cadmium concentration of $\sim 4.4 \mathrm{~g} / \mathrm{L}$ (0.3 absorbance at $350 \mathrm{~nm}$ ), from which $1 \mathrm{~mL}$ is mixed in $300 \mathrm{mg}$ of PS. The weight concentration for this sample is $\sim 1 \%$ and the overall thickness of the $\mathrm{CdSe} / \mathrm{CdS}$ nanocomposite is $\sim 0.5 \mathrm{~mm}$.

The decay kinetics of CdSe/CdS@PS 1\% weight concentration nanocomposite with an exceptional time resolution of $38 \mathrm{ps} \mathrm{FWHM} \mathrm{was} \mathrm{studied}$ during feasibility test beamtime at FemtoMAX ${ }^{44}$ short-pulse facility (SPF at MAX-IV laboratory, Lund, Sweden). The beamline provided $10 \mathrm{keV}$ monochromatic X-ray pulses of $100 \mathrm{fs}$ width (FWHM) at $2 \mathrm{~Hz}$. The scintillation photons were detected by Hamamatsu R3809U-50 MCP-PMT through SCHOTT GG475 long-pass filter. The MCP-PMT operated in a single photon counting mode with a transit time spread of 25 ps FWHM (according to the datasheet). The output of the detector was boosted by an SHF 100 APP broadband preamplifier $(12 \mathrm{GHz}, 19 \mathrm{~dB})$ and digitized by the Lecroy Labmaster 10-36Zi oscilloscope $(36 \mathrm{GHz}, 80 \mathrm{G} \mathrm{s} / \mathrm{s})$. The trigger for the oscilloscope was a signal induced in the RF antenna by electron beam passing the SPF undulator.

After each excitation pulse a waveform containing several single photon peaks correlated with the trigger event was obtained. Each waveform was processed in LabView by its built-in Peak Detector Virtual Instrument, which detected photon time stamps. The decay curves were obtained by accumulating those time stamps over 6-12 hour period, and plotting them on a histogram with $12.5 \mathrm{ps}$ bin width (equal to oscilloscope sampling period). For the determination of IRF of a system we recorded the decay of IBL in $\mathrm{Li}_{2} \mathrm{MoO}_{4}$ crystal using SCHOTT GG420 long-pass filter, and applied Gaussian fit to the recorded curve. The IBL is a prompt weak emission ${ }^{37}$ with decay times on the order of $1 \mathrm{ps}$, and, in all experiments known to us, its time profile repeated the IRF of the system. The luminescence of air having decay time of $\sim 0.5 \mathrm{~ns}$ was suppressed by the long-pass filters used, as it was shown to have negligible intensity at wavelengths longer than $420 \mathrm{~nm}$.

\section{Light yield measurements under X-ray excitation}

The light output study is done by comparing the emission from nanocomposites to the light collected from a LYSO crystal in the same excitation conditions, normalizing to its intrinsic light yield and assuming full energy deposition. All samples are placed in the same position, irradiated with a X-ray beam collimated to $3 \mathrm{~mm}$ diameter and readout using the same integration time window of $500 \mathrm{~ns}$. Measurements were performed in time correlated single photon counting mode using a fast hybrid PMT (HPM-100-07 from Becker\&Hickl) connected through a constant fraction discriminator (ORTEC 9327) to a fast TDC (the xTDC4$\mathrm{PCle}$ from (ronologic). The IRF of the setup has been obtained by recording the time response of IBL present in $\mathrm{Li}_{2} \mathrm{MoO}_{4}$ crystals, which has shown FWHM of $\sim 130 \mathrm{ps}$. For more details, please consider. ${ }^{34}$

$\mathrm{X}$-ray absorption by nano-scintillating layers is used to evaluate the energy deposited by X-rays in the samples under study. For this, we use the Timepix chip from the Medipix collaboration at CERN as X-ray detector. ${ }^{45}$ We monitored the attenuation of the X-ray beam as it passes through the samples and report an attenuation coefficient $\eta$, which it is to correct by the partial energy deposition.

As the X-ray tube energy spectra is not mono-energetic, we use the mean value of the energy deposited in the X-ray detector as a way to compute the energy deposited on the nanocomposite sample. In this regard, we find the mean energy deposited in the sample of interest by subtracting the detector response with and without sample in between the X-ray beam and the detector as it follows:

$$
\eta=\frac{\left\langle E_{d e p}^{N C S}\right\rangle}{\left\langle E_{d e p}^{T P X}\right\rangle}=\frac{\left\langle E_{d e p}^{T P X}\right\rangle-\left\langle E_{d e p}^{T P X}\right\rangle^{N C s}}{\left\langle E_{d e p}^{T P X}\right\rangle}
$$

Here $\left\langle E_{d e p}^{T P X}\right\rangle^{N C S}$ is the mean energy deposited in the Timepix detector when the nanocomposite is in place.

\section{Light yield measurements under electron excitation}

We use a spectral-time-resolved cathodoluminescence setup with a broad energy electron beam and $E_{e}^{\max }=120 \mathrm{keV}$ (with penetration depth of $\sim 0.2 \mathrm{~mm}$ ) to probe the energy transfer mechanisms within CdSe-based nanocomposites.

The spectra and decays were obtained at the pulsed cathodoluminescence setup described in ref. ${ }^{11}$ The electron beam with 200 ps FWHM pulse and peak electron current of $10 \mathrm{~A} / \mathrm{cm}^{2}$ was used as source of excitation. The Andor iStar iCCD was used to obtain the gated spectra in different time windows. For decay curves, a Hamamatsu R3809U-50 MCPPMT was used, with a time response of 250 ps FWHM. A total time window for luminescence monitoring after the excitation pulse was $50 \mu \mathrm{s}$. The IRF was determined by measuring the time profile of hot $\mathrm{IBL}$ of $\mathrm{PbF}_{2}$ single crystal. The samples of CdSe/CdS@PS and LYSO:Ce were installed on the sample holder, and covered by identical aluminum masks to expose the same sample surface area $\left(\sim 4 \times 8 \mathrm{~mm}^{2}\right)$ to the wide homogeneous electron beam. The samples could be positioned in front of the beam by the means of the manipulator, which enables precise comparison of the sample light output in the same conditions.

\section{DATA AVAILABILITY}

The data sets generated during and/or analyzed during the current study are available from the corresponding author on reasonable request.

Received: 17 April 2019; Accepted: 10 September 2019; Published online: 04 October 2019

\section{REFERENCES}

1. The 10 ps challenge, a step toward reconstruction-less TOF-PET. [Online]. Available: https://the10ps-challenge.org.

2. Lecoq, P. Pushing the limits in Time-of-Flight PET imaging. IEEE Trans. Radiat. Plasma Med. Sci. 1, 473-485 (2017).

3. Biograph vision. [Online]. Available: https://www.siemens-healthineers.com/ molecular-imaging/pet-ct/biograph-vision. 
4. Gundacker, S., Auffray, E., Pauwels, K. \& Lecoq, P. Measurement of intrinsic rise times for various $L(Y) S O$ and LuAG scintillators with a general study of prompt photons to achieve 10 ps in TOF-PET. Phys. Med. Biol. 61, 2802 (2016).

5. Nikl, M. \& Yoshikawa, A. Recent R\&D trends in inorganic single-crystal scintillator materials for radiation detection. Adv. Opt. Mater. 3, 463-481 (2015).

6. Dujardin, C. et al. Needs, trends, and advances in inorganic scintillators. IEEE Trans. Nucl. Sci. 65, 1977-1997 (2018).

7. Gundacker, S., Turtos, R. M., Auffray, E., Paganoni, M. \& Lecoq, P. High-frequency SiPM readout advances measured coincidence time resolution limits in TOF-PET. Phys. Med. Biol. 64, 055012 (2019).

8. Korpar, S., Dolenec, R., Križan, P., Pestotnik, R. \& Stanovnik, A. Study of TOF-PET using cherenkov light. Nucl. Instrum. Methods Phys. Sect. A, Accel. Spectrom. Detect. Assoc. Equip. 654, 532-538 (2011).

9. Brunner, S. E., Gruber, L., Marton, J., Suzuki, K. \& Hirtl, A. Studies on the Cherenkov effect for improved time resolution of TOF-PET. IEEE Trans. Nucl. Sci. 61, 443-447 (2014).

10. Brunner, S. E. \& Schaart, D. R. BGO as a hybrid scintillator/cherenkov radiator for cost-effective time-of-flight PET. Phys. Med. Biol. 62, 4421-4439 (2017).

11. Omelkov, S., Nagirnyi, V., Vasil'ev, A. \& Kirm, M. New features of hot intraband luminescence for fast timing. J. Lumin. 176, 309-317 (2016).

12. R. M. Turtos, S. Gundacker, E. Auffray \& P. Lecoq. Towards a metamaterial approach for fast timing in PET: experimental proof-of-concept. Phys. Med. Biol. 64, 185018 (2019).

13. Turtos, R. M. et al. Ultrafast emission from colloidal nanocrystals under pulsed $x$ ray excitation. J. Instrum. 11, P10015 (2016).

14. Achtstein, A. W. et al. Electronic structure and exciton-phonon interaction in twodimensional colloidal CdSe nanosheets. Nano Lett. 12, 3151-3157 (2012).

15. Ithurria, S., Tessier, M. D., Mahler, B., Lobo, R. P. S. M. \& Efros, B. D. A. L. Colloida nanoplatelets with two-dimensional electronic structure. Nat. Mater. 10, 936 (2011).

16. Naeem, A. et al. Giant exciton oscillator strength and radiatively limited dephasing in two-dimensional platelets. Phys. Rev. B 91, 121302 (2015).

17. Grim, J. Q. et al. Continuous-wave biexciton lasing at room temperature using solution-processed quantum wells. Nat. Nanotechnol. 9, 891-895 (2014)

18. Woggon, U. et al. Huge binding energy of localized biexcitons in CdS/ZnS quantum structures. Phys. Rev. B 61, 126'32-12635 (2000).

19. Lecoq, P. Development of new scintillators for medical applications. Nucl. Instrum. Methods Phys. Res.Sect. A, Accel. Spectrom. Detect. Assoc. Equip. 809, 130-139 (2016).

20. Bulin, A.-L. et al. Modelling energy deposition in nanoscintillators to predict the efficiency of the x-ray-induced photodynamic effect. Nanoscale 7, 5744-5751 (2015).

21. Padilha, L. A., Bae, W. K., Klimov, V. I., Pietryga, J. M. \& Schaller, R. D. Response of semiconductor nanocrystals to extremely energetic excitation. Nano Lett. 13 925-932 (2013)

22. Burešová, $\mathrm{H}$. et al. Preparation and luminescence properties of $\mathrm{ZnO}: \mathrm{Ga}$ - polystyrene composite scintillator. Opt. Express 24, 15289-15298 (2016).

23. Liu, C. et al. Facile single-precursor synthesis and surface modification of hafnium oxide nanoparticles for nanocomposite gamma-ray scintillators. Adv. Funct. Mater. 25, 4607-4616 (2015).

24. Létant, S. E. \& Wang, T.-F. Semiconductor quantum dot scintillation under gamma ray irradiation. Nano Lett. 6, 2877-2880 (2006).

25. Létant, S. E. \& Wang, T.-F. Study of porous glass doped with quantum dots or laser dyes under alpha irradiation. Appl. Phys. Lett. 88, 103110 (2006).

26. Turtos, R. M. et al. Timing performance of $\mathrm{ZnO}: \mathrm{Ga}$ nanopowder composite scintillators. Physica. Status Solidi (RRL) 10, 843-847 (2016).

27. Bertrand, G. H. V., Polovitsyn, A., Christodoulou, S., Khan, A. H. \& Moreels, I. Shape control of zincblende CdSe nanoplatelets. Chem. Commun. 52, 11 975-11978 (2016).

28. Antanovich, A., Prudnikau, A., Matsukovich, A., Achtstein, A. \& Artemyev, M. Selfassembly of CdSe nanoplatelets into stacks of controlled size induced by ligand exchange. J. Phys. Chem. C. 120, 5764-5775 (2016).

29. Donegá, Cd. M. Synthesis and properties of colloidal heteronanocrystals. Chem. Soc. Rev. 40, 1512-1546 (2011).

30. Yadav, S., Singh, A., Thulasidharan, L. \& Sapra, S. Surface decides the photoluminescence of colloidal CdSe nanoplatelets based core/shell heterostructures. J. Phys. Chem. C. 122, 820-829 (2018).

31. Liu, C. et al. Transparent ultra-high-loading quantum dot/polymer nanocomposite monolith for gamma scintillation. ACS Nano 11, 6422-6430 (2017).

32. Rabouw, F. T. et al. Temporary charge carrier separation dominates the photoluminescence decay dynamics of colloidal CdSe nanoplatelets. Nano Lett. 16 2047-2053 (2016).

33. Turtos, R. M. et al. Measurement of LYSO intrinsic light yield using electron excitation. IEEE Trans. Nucl. Sci. 63, 475-479 (2016)
34. Turtos, R. M., Gundacker, S., Omelkov, S., Auffray, E. \& Lecoq, P. Light yield of scintillating nanocrystals under $x$-ray and electron excitation. J. Lumin. 215 116613 (2019).

35. Gundacker, S., Turtos, R., Auffray, E. \& Lecoq, P. Precise rise and decay time measurements of inorganic scintillators by means of x-ray and 511 kev excitation. Nucl. Instrum. Methods Phys. Res. Sect. A, Accel. Spectrom. Detect. Assoc. Equip. 891 42-52 (2018)

36. Piemonte, C. et al. Performance of NUV-HD silicon photomultiplier technology. IEEE Trans. Electron Devices 63, 1111-1116 (2016).

37. Omelkov, S. I. et al. Scintillation yield of hot intraband luminescence. J. Lumin. 198, 260-271 (2018)

38. Wanarak, C., Chewpraditkul, W. \& Phunpueok, A. Light yield non-proportionality and energy resolution of Lu1.95Y0.05SiO5:Ce and Lu2SiO5:Ce scintillation crystals. Procedia Eng. 32, 765-771 (2012).

39. Rowland, C. E. et al. Picosecond energy transfer and multiexciton transfer outpaces Auger recombination in binary CdSe nanoplatelet solids. Nat. Mater. 14 484-489 (2015).

40. Agostinelli, S. et al. Geant4-a simulation toolkit. Nucl. Instrum. Methods Phys. Res., Sect. A, Accel. Spectrom. Detect. Assoc. Equip. 506, 250-303 (2003).

41. Allison, J. et al. Geant4 developments and applications. IEEE Trans. Nucl. Sci. 53 270-278 (2006).

42. Tessier, M. D. et al. Efficient exciton concentrators built from colloidal core/crown CdSe/CdS semiconductor nanoplatelets. Nano Lett. 14, 207-213 (2014).

43. Cates, J. W., Gundacker, S., Auffray, E., Lecoq, P. \& Levin, C. S. Improved single photon time resolution for analog SiPMs with front end readout that reduces influence of electronic noise. Phys. Med. Biol. 63, 185022 (2018).

44. Enquist, $H$. et al. FemtoMAX - an x-ray beamline for structural dynamics at the short-pulse facility of MAX IV. J. Synchrotron Radiat. 25, 570-579 (2018).

45. Poikela, T. et al. Timepix3: a $65 \mathrm{~K}$ channel hybrid pixel readout chip with simultaneous ToA/ToT and sparse readout. J. Instrum. 9, C05013-C05013 (2014).

\section{ACKNOWLEDGEMENTS}

This work was supported by the ERC Advanced Grant no. 338953 (TICAL), the ERC Starting Grant no. 714876 (PHOCONA), and Estonian Research Council (projects PUT1081, IUT2-26). A partial financial support from the Estonian Centre of Excellence TK141 by the EU through the European Regional Development Fund (TK141, project no. 2014-2020.4.01.15-0011) is gratefully acknowledged. The research have been developed in the frame of the Crystal Clear Collaboration and the Cost Action FAST TD1401. The authors are grateful to MAX-IV laboratory (Lund, Sweden) and FemtoMAX team lead by J. Larsson for the opportunity of scintillation decay experiment at 100-fs X-ray excitation. We are also most thankful for the collaboration maintained with the Medipix group at CERN, specially for the help of Lukas Tlustos and Jerome Alozy regarding technical support and the energy calibration of the Timepix detector.

\section{AUTHOR CONTRIBUTIONS}

R.M.T, S.G., I.M., E.A. and P.L, as a team, conceived the idea of implementing CdSe nanoplatelets in combination with standard scintillators for ultrafast timing applications. R.M.T. performed the characterization and drop-casting of the $\mathrm{CdSe} /$ CdS films on LYSO, the assembly of the CdSe-LYSO sampling pixel, the light yield measurements of CdSe-based nanocomposite and data analysis for most of the results. R.M.T. wrote the manuscript with contributions from all authors. S.G. designed the readout and performed the first CTR and rise time measurements of CdSe + LYSO pixel with $511 \mathrm{keV}$ gammas. S.O. installed the TCSPC setup allowing for light yield measurements with $\mathrm{X}$-rays and performed all cathodoluminescence experiments. As well, S.O, J.S., and M.K. performed the kinematic measurements at FemtoMAX. B.M. synthesized the $\mathrm{CdSe} / \mathrm{CdS}$ used in the study and proceed with the solution treatment to obtain dry drop-casted films and the embedding procedure in PS. A.K. know-how was critical in the preparation of the CdSe in PS sample. C.D. and Z.M. characterized highly diluted $\mathrm{CdSe} / \mathrm{CdS}$ films under different laser powers and Xray excitation. A.V. produced and wrote all the theoretical considerations presented here.

\section{COMPETING INTERESTS}

The authors declare no competing interests.

\section{ADDITIONAL INFORMATION}

Supplementary information is available for this paper at https://doi.org/10.1038/ s41699-019-0120-8. 
Correspondence and requests for materials should be addressed to R.M.T.

Reprints and permission information is available at http://www.nature.com/ reprints

Publisher's note Springer Nature remains neutral with regard to jurisdictional claims in published maps and institutional affiliations.

Open Access This article is licensed under a Creative Commons (c) Attribution 4.0 International License, which permits use, sharing,
adaptation, distribution and reproduction in any medium or format, as long as you give appropriate credit to the original author(s) and the source, provide a link to the Creative Commons license, and indicate if changes were made. The images or other third party material in this article are included in the article's Creative Commons license, unless indicated otherwise in a credit line to the material. If material is not included in the article's Creative Commons license and your intended use is not permitted by statutory regulation or exceeds the permitted use, you will need to obtain permission directly from the copyright holder. To view a copy of this license, visit http://creativecommons. org/licenses/by/4.0/.

(c) The Author(s) 2019 